Der weltweite Ausstieg von BSH aus der FCKW- und FKW-Technologie als Beispiel für die Verbreitung umwelffreundlicher Technologien in globalen Märkten

\section{Unternehmen setzen Standards}

\author{
Wenn global agierende Unternehmen einen konkreten Beitrag zu einer \\ nachhaltigen Entwicklung leisten wollen, muß sich ihr Handeln weltweit als \\ umwelt- und sozialverträglich erweisen. Zum Unternehmenskonzept der BSH \\ Bosch und Siemens Hausgeräte $\mathrm{GmbH}$ gehört es daher, hier bereits etablierte \\ Umweltschutzstandards auch im Ausland einzuhalten und so zu deren \\ Verbreitung auf den globalen Märkten beizutragen.
}

$\mathrm{A}$ Von Dieter Bärmann Is weltweit agierendes Unternehmen und Nummer vier der Branche ist die BSH aktiv an den Entwicklungsprozessen der sogenannten ,Emerging Markets' beteiligt. Hierzu gehört vornehmlich der Know-How-Transfer in diese Regionen. Bei der Planung und der Errichtung neuer Werke im Ausland werden alle hier etablierten Umweltschutztechnologien umgesetzt, bereits bestehende Standorte werden entsprechend umgerüstet. Die Produkte entsprechen - unabhängig vom Herstellungsort - höchsten Umweltstandards. Parallel dazu muß aber auch Verständnis dafür geweckt werden, daß wirtschaftliches Handeln in den meisten Fällen ökologische Folgen mit häufig globalen Auswirkungen hat. Die BSH leistet auch hier ihren Beitrag.

Die fortschreitende Erwärmung der Erdatmosphäre ist dafür das markanteste Beispiel. Sie hat

\section{Umwelt und Innovation}

ist das Thema des Informationsdienstes

Ökologisches

Wirtschaften 2/99

\section{Mit Öko-Effizienz zur} Nachbaltigkeit?

ist das Thema des Informationsdienstes

$$
\begin{gathered}
\text { Ökologisches } \\
\text { Wirtschaften 3/99 }
\end{gathered}
$$

Wenn Sie potentielle Beiträge haben, wenden Sie sich bitte an die Redaktion. eine primäre Ursache: das Einbringen von treibhausrelevanten Gasen in die Atmosphäre. Dazu gehören unter anderem Kohlendioxid, das bei vielen Verbrennungsprozessen entsteht, aber auch die Freisetzung ,künstlicher' Gase wie Fluorchlorkohlenwasserstoffe (FCKW) und Fluorkohlenwasserstoffe (FKW), beides Derivate der in der Natur vorkommenden Kohlenwasserstoffe. Neben der Treibhausrelevanz dieser künstlichen Gase kommt bei den FCKWs, deren Mengeneinsatz in den fünfziger Jahren begann, ein Ozonzerstörungspotential hinzu.

\section{Hausgeräte und Klimaschutz}

Beide Effekte - die Erwärmung der Erdatmosphäre und die Zerstörung der Ozonschicht sind so gravierend, daß sie zu einem Umsteuern zwingen, und zwar konsequent und weltweit. Dem Schutz der Ozonschicht dient letztlich nur der völlige Verzicht auf FCKWs, also deren Ersatz durch reine Kohlenwasserstoffe. Das gilt auch für die sogenannten weichen FCKWs, die verglichen mit den harten FCKWs immerhin noch ein Ozonschädigungspotential von zehn bis zwanzig Prozent besitzen. Eine Verringerung des Treibhauseffekts läßt sich wiederum nur durch die Senkung des Energieverbrauchs, also des Kohlendioxidausstosses, erreichen. In beiden Fällen ist die Hausgeräteindustrie gefordert: Einerseits kommen bei der Kühlung und Isolierung von Kühl- und Gefriergeräten FCKW und FKW zum Einsatz. Zum zweiten trägt der Betrieb von Haushaltsgeräten wie Waschmaschinen, Kühlschränken und Geschirrspülern maßgeblich zum Energieverbrauch des privaten Sektors bei.

Was die FCKWs in Kühl- und Gefriergeräten angeht, so sind sie in Europa seit 1996 verboten. In den USA werden dagegen weiterhin weiche FCKWs eingesetzt. In China, Südostasien und Südamerika kommen sowohl harte als auch weiche FCKWs zum Einsatz. Gleiches gilt weitgehend für Afrika. Das Abkommen von Montreal, das den weltweiten Ausstieg aus der FCKW-Technologie regelt, sieht allerdings vor, den Entwicklungs- und Schwellenländern eine Frist bis zum Jahr 2010 einzuräumen.

\section{- Weltweiter Umstieg}

Bereits 1988 konnte die BSH als erster Hausgerätehersteller der Welt die in den Geräten eingesetzte FCKW-Menge halbieren. Um den Ausstieg zu realisieren, folgte sorgfältige Entwicklungsarbeit. Selbst die gesetzlichen Grundlagen für brennbare Kältemittel mußten erst noch unter Beteiligung der Wirtschaft geschaffen werden. Auf dieser Basis erarbeitete die BSH zusammen mit dem Technischen Überwachungsverein (TÜV) die Sicherheitsvorschriften, die im Grundsatz heute weltweit Maßstab für Produktion und die Produkttechnologie der Hausgerätebranche sind.

Im Jahr 1993 begann die BSH dann, die Kältegeräte in Deutschland umzustellen. Bereits ein Jahr später konnten alle europäischen BSH-Standorte auf den Einsatz von FCKWs verzichten. Der nächste Schritt, der vollständige Verzicht auf FKWs und damit die weltweite Umstellung auf die Kohlenwasserstofftechnologie, scheiterte zunächst an der mangelnden Verfïgbarkeit von Kompressoren. Dies ist übrigens ein Grund, weshalb sich die Umstellung bei Foron schneller realisieren ließ. Der Kühlgerätehersteller, der dabei 1992 von der Umweltorganisation Greenpeace unterstiitzt wurde, besaß eine eigene Kompressorenproduktion und benötigte vergleichsweise kleine Stïckzahlen. Die BSH brauchte dagegen rund 4,8 Millionen Verdichter jährlich. Gemeinsam mit dem deutschen Zulieferer Danfoss begann die BSH deshalb im selben Jahr, die Verdichter auf Kohlenwasserstofftechnologie umzustellen, ein Prozeß, der Mitte 1993 abgeschlossen war.

Mittlerweile wurden alle BSH-Zulieferer weltweit dazu verpflichtet, Kompressoren in Kohlenwasserstofftechnik bereitzustellen. Die BSH ist deshalb nun in der Lage, in den kommenden zwei Jahren den kompletten Umstieg auf Kohlenwasserstofftechnologie zu realisieren. So wird das Kältegerätewerk in China ab Frühjahr 1999 die ersten Kühl- und Gefriergeräte in Kohlenwasserstofftechnologie produzieren. Das Werk in Brasilien folgt einige Monate später.

Der Umstieg der BSH auf die Kohlenwasserstofftechnologie, den die Umweltorganisation Greenpeace weltweit mit ihren Organisationen vor Ort 
unterstüzt, ist technisch anspruchsvoll und bedarf auch hoher Investitionen. Allein in Deutschland wurden für Grundlagenforschung, sicherheits- und produktionstechnische Entwicklung sowie Installation der Anlagen rund 20 Millionen Mark investiert. Einen Betrag gleicher Größenordnung beansprucht die Umstellung der Kältegerätewerke in Spanien, Griechenland, China, Brasilien und der Türkei. Grundsätzlich umfaßt das Umstiegsszenario bis zum Jahr 2000 folgende Maßnahmen:

- Um die Geräte weltweit so zu konstruieren, daß der ausschließliche Einsatz von Kohlenwasserstoffen möglich ist, wird ein weltweit verbindlicher technischer Standard erarbeitet.

- Beim Neubau von Fertigungsstätten wird die Kohlenwasserstofftechnologie bereits bei der Planung des Herstellungsprozesses integriert.

- Standorte, die übernommen wurden, werden nach dem neuen Standard umgerïstet.

\section{Umweltschutz im Wettbewerb}

Diese konsequent umweltgerechte Strategie der BSH hat über den weltweiten Wettbewerb auch umweltverträgliches Verhalten in Ländern bewirkt, die sich der neuen Technik bisher verweigert haben. So stellten mittlerweile alle Hersteller in Japan, die sich traditionell eher stark an den USA orientieren, auf die BSH-Technik im Isolationsbereich um. Gleiche Erfahrungen ließen sich in Brasilien machen, wo bis zum Markteintritt der BSH ausschließlich in FCKWTechnologie produziert wurde. Nachdem die BSH dort für ihre neuen Produkte zahlreiche Umweltauszeichnungen erhalten und damit einen nachhaltigen Markterfolg erzielt hatte, stellten die Wettbewerber sukzessive auf die umweltverträgliche Technik um. Anfragen zum Technologietransfer liegen mittlerweile auch aus den USA vor.

Für den Klimaschutz von Bedeutung ist auch die Entwicklung energiesparender Geräte. Allein in Deutschland sind derzeit rund 60 Millionen Kühl- und Gefriergeräte Tag und Nacht in Betrieb. Ihr Anteil am Gesamtenergieverbrauch ist mit rund fünf Prozent zwar vergleichsweise gering. Doch würden all diese Geräte durch Neugeräte mit einem dreißigprozentigen Energieeinsparungspotential ersetzt, so könnte man immerhin ein Kraftwerk abschalten. Noch wichtiger ist der Einsatz von energiesparenden Kühlgeräten in den Entwicklungsländern, da diese in der Regel keine ausreichende Energieversorgung besitzen.

\section{- Marktvorteil durch Verbrauchsreduktion}

Daß die BSH auch an die Verbrauchwerte ihrer Geräte weltweit denselben Maßstab anlegt, wird deshalb sehr wohl von den ,Emerging Markets' honoriert. Bereits jetzt wird beispielsweise in China beim Kauf von Hausgeräten großer Wert auf niedrige Verbrauchswerte gelegt, da Energie dort vergleichsweise teuer ist. Mit verbrauchsarmen Geräte zu attraktiven Preisen besitzt die BSH einen deutlichen Marktvorteil. Die Bedeutung energiesparender Lösungen wird sogar noch steigen, wenn die chinesische Regierung 1999 eine Verbrauchskennzeichnung einführt, der dasselbe System zugrundeliegt wie dem europäischen Energielabel.

Indem die BSH ihr hohes Umweltschutzniveau bei Produktion und Produkten an allen Standorten etabliert und Erfahrungen weitergibt, trägt sie zum globalen Umweltschutz und damit zu einer nachhaltigen Entwicklung bei. So kann auch nur ein weltweit agierendes Unternehmen wie die BSH der umweltverträglichen Kohlenwasserstofftechnologie zum Durchbruch verhelfen: „Mit ihren Bemühungen hat die BSH sowohl in Deutschland als auch durch ihr vielfältiges Enga gement im Ausland erheblich dazu beigetragen, daß umweltschädliche Kältemittel in die Defensive geraten und sich umwelffreundliche - also ozonschichtschonende, klimaneutrale und energiesparende - Geräte weltweit am Markt durchsetzen“, so Wolfgang Lohbeck von Greenpeace Deutschland, der in regelmäßigem Austausch mit der Entwicklungsabteilung in Giengen steht.

\section{Der Autor}

Dieter Bärmann ist Leiter des Produktbereichs, Kälte' der BSH Bosch und Siemens Hausgeräłe GmbH. Kontakt: BSH, Robert-Bosch-Straße, 89537 Giengen. Tel. $07322 / 9222$ 14, Fax $07322-923504$ E-mail: Dieter.Baermann@bshg.com

\section{Beilagenhinweis}

\section{Unsere Publikation enthält einen Prospekt des VablenVerlages.}

Wir bitten um freundliche Beachtung.
Zum Thema

Sechsmal im Jahr: Die Fachzeitschrift für Umweltrecht -

und damit sechsmal im Jahr ein kompletter Überblick über das Umweltrecht.

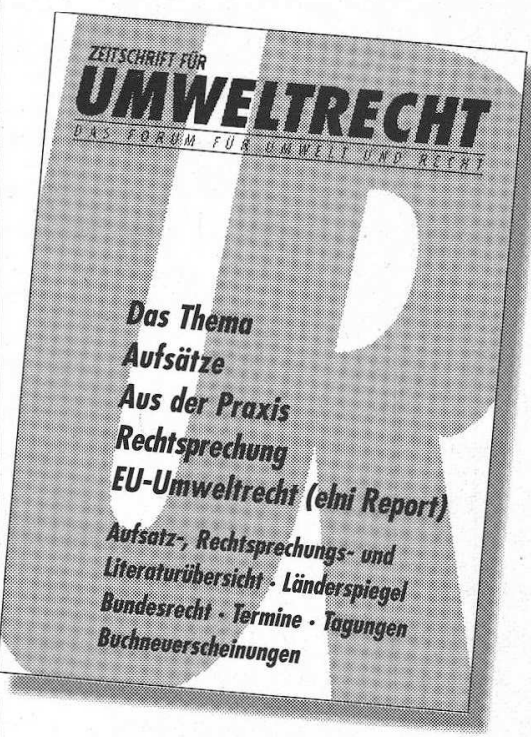

\section{Aktuelle wissenschaftliche Beiträge und Analysen}

- diskutieren Stand und Entwicklung des Umweltrechts.

\section{Ein umfangreicher Service-Teil}

- bringt die neueste Rechtsprechung,

- informiert über die aktuelle Gesetzesentwicklung auf Landes-, Bundes- und Europaebene und

- dokumentiert Aufsätze aus über einhundert Fachzeitschriften sowie wichtige Nachrichten, Termine und Buchneuerscheinungen.

Zu bestellen bei: Rhombos-Verlag Kurfürstenstr. 17, 10785 Berlin Tel. 030/2619461, Fax 030/2616854 
(c) 20I0 Authors; licensee IÖW and oekom verlag. This is an article distributed under the terms of the Creative Commons Attribution Non-Commercial No Derivates License (http://creativecommons.org/licenses/by-nc-nd/3.o/), which permits unrestricted use, distribution, and reproduction in any medium, provided the original work is properly cited. 